\title{
Clustering and Halogen Effects Enabled Red/Near-Infrared Room Temperature Phosphorescence from Aliphatic Cyclic Imides
}

\author{
Tianwen Zhu, Tianjia Yang, Qiang Zhang, and Wang Zhang Yuan*
}

School of Chemistry and Chemical Engineering, Frontiers Science Center for Transformative Molecules, Shanghai Key Lab of Electrical Insulation and Thermal Aging, Shanghai Jiao Tong University, No. 800 Dongchuan Rd., Minhang District, Shanghai 200240, China

E-mail:wzhyuan@sjtu.edu.cn

\begin{abstract}
Pure organics with room temperature phosphorescence (RTP) are urgently demanded in advanced optoelectronic and bioelectronic applications. However, currently reported phosphors are mostly aromatics and restricted to blue to orange colors. It remains an enormous challenge to achieve red and near-infrared (NIR) RTP, particularly for those from nonaromatics. Here we demonstrate a series of cyclic imides derived from succinimide, which can emit red $(665,690 \mathrm{~nm})$ and even NIR (745 nm) RTP with outstanding efficiencies of up to $9.2 \%$, despite their rather limited molecular conjugations. Such unique emission should be ascribed to the presence of the imide unit and heavy atoms, effective molecular clustering, and the electron delocalization of halogens, which not only greatly facilitate intersystem crossing, but also afford significantly extended through-space conjugation and rigidified conformations. These results pave the way to the rational construction of red and NIR nonconventional luminophores through synergistic clustering and halogen effects.
\end{abstract}

Keywords: cyclic imides, NIR phosphorescence, though-space conjugation, clustering-triggered emission, halogen effect 
Pure organic materials with efficient room temperature phosphorescence (RTP) have aroused tremendous interest due to their unique photophysical properties, ${ }^{[1]}$ facile synthetic procedures, low cost, structural designability, and extensive applications in organic light-emitting diodes, ${ }^{[2]}$ bioimaging, ${ }^{[3]}$ sensing, ${ }^{[4]}$ information security, ${ }^{[5]}$ and so forth. Currently, most reported pure organic RTP luminophores are aromatic, whose emission colors generally range from blue to orange, ${ }^{[1-7]}$ with scattered red and nearinfrared (NIR) examples. ${ }^{[8,9]}$ Particularly, the access of NIR RTP is the most difficult, owing to the nonradiative deactivations caused by the inherent low band gaps between $\mathrm{T}_{1}$ and $\mathrm{S}_{0}$ states. ${ }^{[10]}$ Recently, in parallel to aromatic luminogens, emerging nonconventional luminophores free of large conjugated moieties are found to emit distinct RTP. ${ }^{[11-13]}$ Their RTP colors, however, are predominated by blue, green, and yellow, ${ }^{[11-14]}$ much redder emissions are hardly found, owing to the preliminary mechanism understanding and consequent lack of rational guide for the photoluminescence (PL) modulation. ${ }^{[15]}$ Construction of nonconventional luminophores with red and NIR RTP are thus of crucial importance for a better understanding of the luminescent mechanism and for the exploration of potential optoelectronic and biological applications. ${ }^{[15]}$

To acquire efficient RTP from nonconventional luminophores, the clustering of functional units, regular molecular packing, and effective intra/intermolecular interactions play critical roles, ${ }^{[14]}$ which are highly associated with the clustering-triggered emission (CTE) mechanism. ${ }^{[11,15 \mathrm{~d}, 16]}$ How to achieve red/NIR RTP, however, remains unclear and underexplored. Herein, we report our recent endeavors in the fabrication of such nonconventional luminophores with bright red/NIR RTP. A group of halogenated cyclic imides derived from succinimide (SI), namely trans-2,3-dibromosuccinimide (DBSI), 2,3dibromomaleimide (DBMI), and 2,3-diiodomaleimide (DIMI) (Figure 1a, S1, and S2), were prepared and investigated. The adoption of such heterocycles is based on the following considerations: firstly, the presence of carbonyl, nitrogen, and halogen moieties could promote spin-orbit coupling (SOC) and intersystem crossing (ISC) transitions, which are significant to generate triplets; secondly, the imide group is expected to form effective intermolecular interactions to stiffen the molecular conformations and 
moreover to generate effective through-space conjugation (TSC); thirdly, the planar cycle is beneficial to form close $\pi$ - $\pi$ stacking and to lower the bandgaps of the aggregates, ${ }^{[17]}$ thus favoring for much redder emissions.

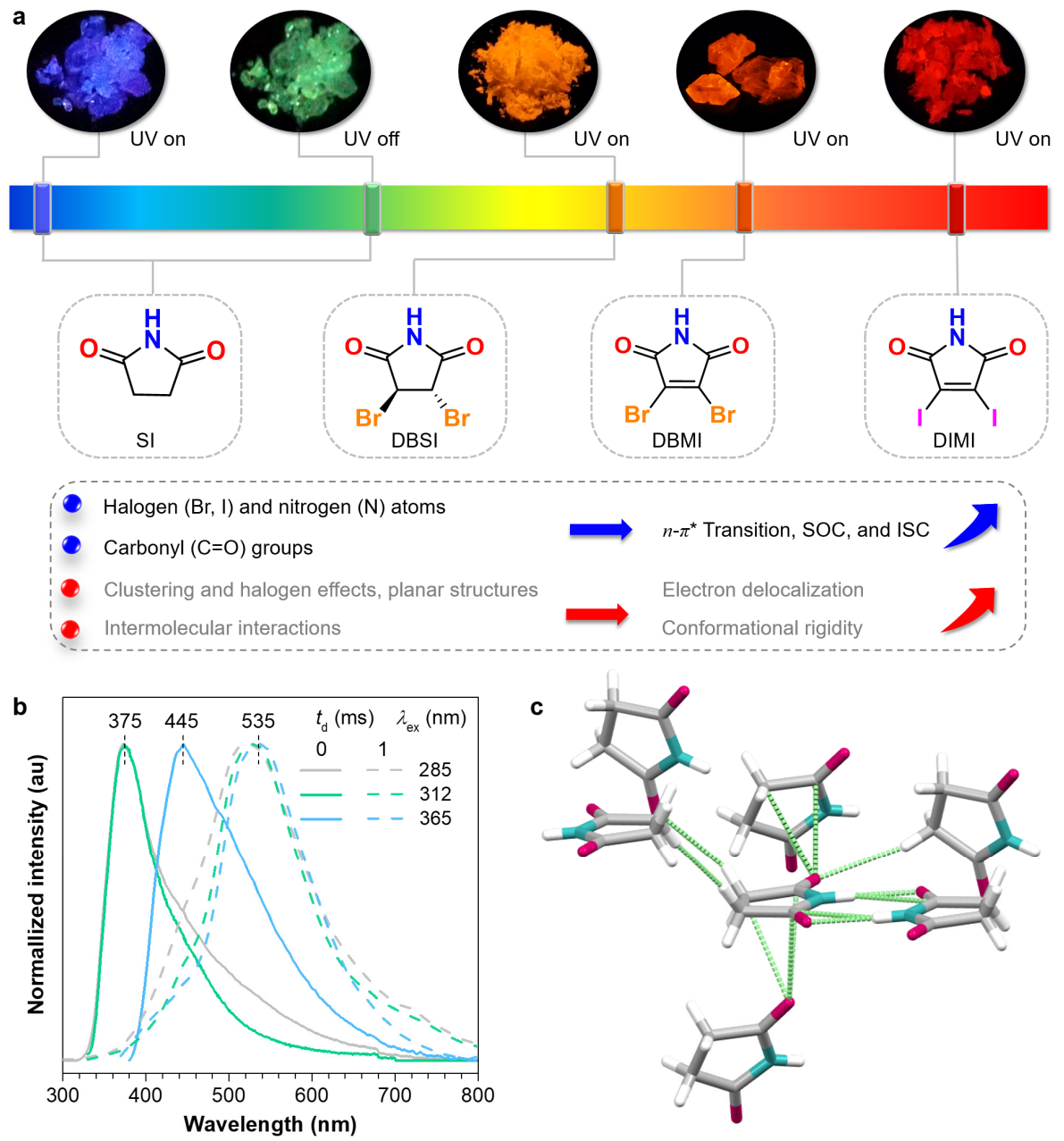

Figure 1. (a) Structure, luminescent photographs of the crystals, and design considerations of SI, DBSI, DBMI, and DIMI. (b) Prompt and delayed emission spectra of SI crystals. (c) Single crystal structure, fragmental molecular packing, and intermolecular interactions around one molecule of SI.

Particularly, SI crystals display excitation-dependent emission peaking at 375 or $445 \mathrm{~nm}$, accompanying intense green afterglow at around $535 \mathrm{~nm}$ (Figure 1a,b), whose lifetimes $(<\tau>)$ are 2.15, $3.54 \mathrm{~ns}$, and $536.0 \mathrm{~ms}$ (Figure S3 and Table S1), suggestive of their fluorescence and RTP features, respectively. Such bright dual emission should be ascribed to the effective intermolecular interactions and remarkable TSC (Figure 1c). ${ }^{[12]}$ In contrast, the other crystals emit unexpected bright orange to red PL 
free of afterglows (Figure 1a), which are mainly found to be RTP emissions ranging from orange-red to NIR. To the best of our knowledge, these are first examples of small nonconventional luminophores with explicit structure and molecular packing that demonstrate red or even NIR RTP.
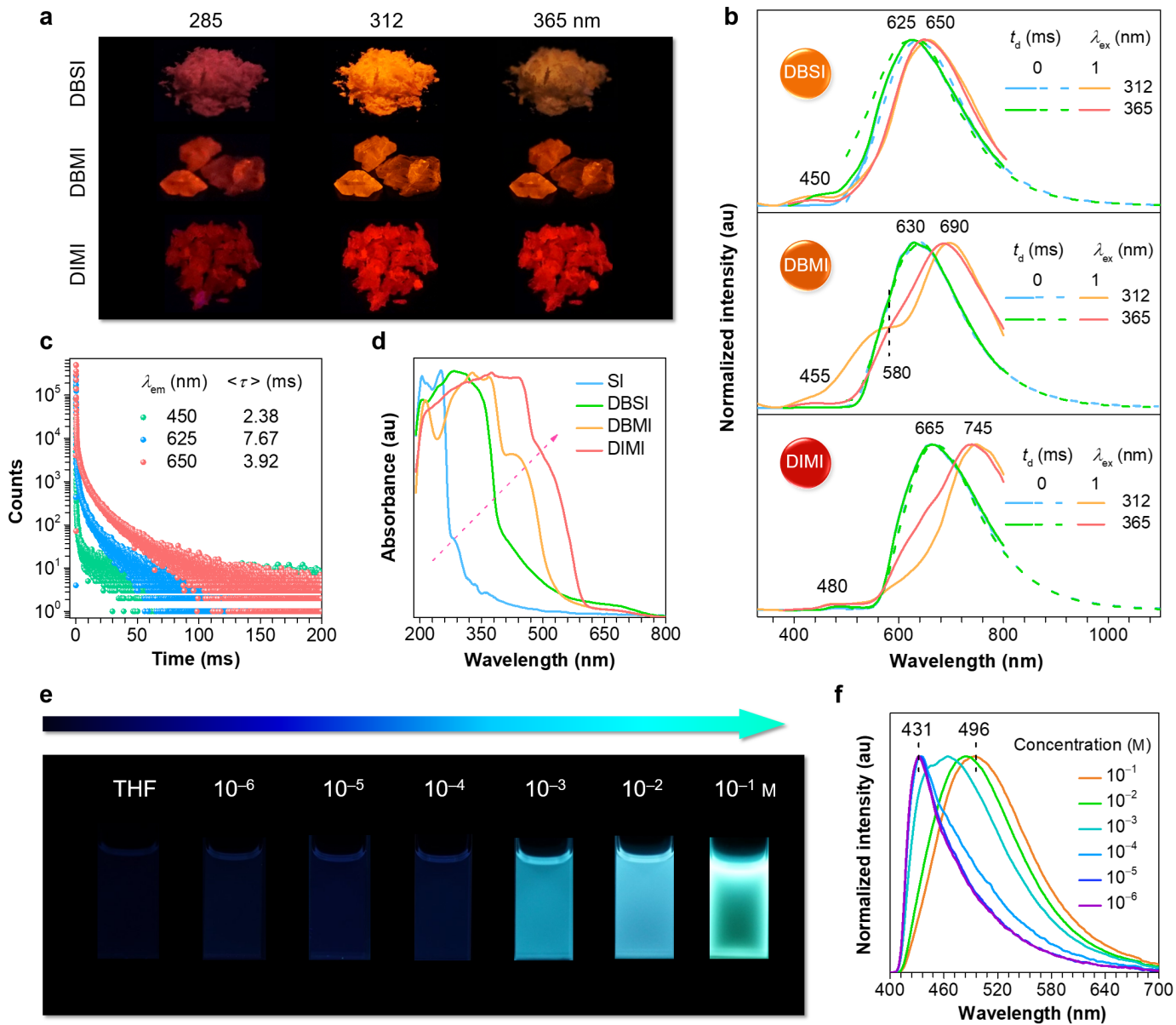

Figure 2. (a) Photographs taken under varying UV lights and (b) prompt $\left(t_{\mathrm{d}}=0 \mathrm{~ms}\right)$ and delayed $\left(t_{\mathrm{d}}=1 \mathrm{~ms}\right)$ emission spectra with varying $\lambda_{\text {exS }}$ of DBSI, DBMI, and DIMI single crystals. ${ }^{[19]}$ (c) Lifetime profiles of DBSI single crystals monitored at different emission wavelengths $\left(\lambda_{\mathrm{ems}}\right)\left(\lambda_{\mathrm{ex}}=312 \mathrm{~nm}\right)$. (d) Absorption spectra of different single crystals. (e) Photographs and (f) normalized emission spectra of varying DBSI/THF solutions with $365 \mathrm{~nm}$ UV irradiation.

Table 1. Dynamic photophysical parameters of the single crystals of SI, DBSI, DBMI, and DIMI. ${ }^{\mathrm{a}}$

\begin{tabular}{|c|c|c|c|c|c|c|c|c|c|c|c|c|c|c|c|}
\hline Sample & $\begin{array}{c}\lambda_{f} \\
{[\mathrm{~nm}]}\end{array}$ & $\begin{array}{c}\lambda_{\mathrm{p} 1} \\
{[\mathrm{~nm}]}\end{array}$ & $\begin{array}{c}\lambda_{\mathrm{p} 2} \\
{[\mathrm{~nm}]}\end{array}$ & $\begin{array}{c}\Phi_{\mathrm{c}} \\
{[\%]}\end{array}$ & $\begin{array}{c}\Phi_{\mathrm{f}} \\
{[\%]}\end{array}$ & $\begin{array}{c}\Phi_{\mathrm{p}} \\
{[\%]}\end{array}$ & $\begin{array}{l}\Phi_{\mathrm{p} 1} \\
{[\%]}\end{array}$ & $\begin{array}{c}\Phi_{\mathrm{p} 2} \\
{[\%]}\end{array}$ & $\begin{array}{l}<\tau_{\mathrm{f}} \\
{[\mathrm{ns}]}\end{array}$ & $\begin{array}{l}\langle\tau\rangle_{\mathrm{pl} 1} \\
{[\mathrm{~ms}]}\end{array}$ & $\begin{array}{l}\langle\tau\rangle_{\mathrm{p} 2} \\
{[\mathrm{~ms}]}\end{array}$ & $\begin{array}{l}k_{r}^{\mathrm{p} 1} \\
{\left[\mathrm{~s}^{-1}\right]}\end{array}$ & $\begin{array}{l}k_{r}^{\mathrm{p} 2} \\
{\left[\mathrm{~s}^{-1}\right]}\end{array}$ & $\begin{array}{l}k_{n r}^{\mathrm{p} 1} \\
{\left[\mathrm{~s}^{-1}\right]}\end{array}$ & $\begin{array}{l}k_{n r}^{\mathrm{p} 2} \\
{\left[\mathrm{~s}^{-1}\right]}\end{array}$ \\
\hline SI & 375 & 535 & - & 16.6 & 9.8 & 6.8 & 6.8 & - & 2.16 & 536.0 & - & 0.1 & - & 1.7 & - \\
\hline DBSI & 450 & 625 & 650 & 4.2 & 0.1 & 4.1 & 1.9 & 2.2 & 2.53 & 7.67 & 3.92 & 2.5 & 5.6 & 127.9 & 249.5 \\
\hline DBMI & 455 & 630 & 690 & 9.2 & $\sim 0$ & 9.2 & 4.0 & 5.2 & $-^{b}$ & 1.38 & 2.86 & 29.0 & 18.2 & 695.6 & 331.5 \\
\hline DIMI & 480 & 665 & 745 & 7.2 & $\sim 0$ & 7.2 & 2.8 & 4.4 & $-_{b}^{b}$ & 3.17 & 1.65 & 8.8 & 26.7 & 306.6 & 579.4 \\
\hline
\end{tabular}

[a] $\lambda_{e x}=312 \mathrm{~nm} ; \Phi_{\mathrm{c}}=\Phi_{\mathrm{f}}+\Phi_{\mathrm{p}} ; k_{r}^{p}=\Phi_{\mathrm{p}} /<\tau>_{\mathrm{p}} ; k_{n r}^{\mathrm{p}}=\left(1-\Phi_{\mathrm{p}}\right) /<\tau>_{\mathrm{p}}$. ${ }^{[\mathrm{b}]}$ Cannot be detected, presumably owing to their tiny fractions. 
Under different UV irradiations, DBSI, DBMI, and DIMI crystals generate orange, orange-red, and deep red emissions (Figure 2a), with maxima at 625, 630, and $665 \mathrm{~nm}$ (Figure 2b and S4a), respectively. Such intriguing orange to red PL from single crystals of small aliphatic heterocycles is rarely observed. ${ }^{[18]}$ These emissions are significantly red-shifted when compared with that of SI crystals, testifying the possibility to access red PL from small cyclic imides through rational molecular and crystal engineering. Distinct to SI crystals, after ceasing the excitations, no afterglow could be observed. Time-resolved measurement with a delay time $\left(t_{\mathrm{d}}\right)$ of $1 \mathrm{~ms}$ shows certain shoulders consistent with the prompt PL; furthermore, emerging peaks at $\sim 650,690$, and $745 \mathrm{~nm}$ are noticed (Figure $2 \mathrm{~b}$ and S4a). To identify these emissions, their $\langle\tau\rangle$ values were monitored. For DBSI crystals, both short (2.53 ns) and long lifetimes $(2.38 \mathrm{~ms})$ are detected at $450 \mathrm{~nm}$ (Figure 2c and S5), indicative of its fluorescence nature; meanwhile, long $\langle\tau\rangle$ S of 7.67 and $3.92 \mathrm{~ms}$ at 625 and $650 \mathrm{~nm}$ were recorded (Figure 2c and Table S2), respectively, illustrating their RTP feature. Notably, for DBMI and DIMI crystals, no distinct ns-scale lifetimes were measured, whereas ms-scale species at different $\lambda_{\text {ems }}$ were detected (Figure S3b and Table S2), which are associated with the RTP emissions. Taken together, these results indicate that the emission of the crystals should be predominantly multiple RTP with varying lifetimes $(<\tau>$ p $)$ and maxima $\left(\lambda_{\mathrm{p}}\right)$.

PL efficiencies of the crystals $\left(\Phi_{\mathrm{c}}\right)$ were further determined and summarized along with other photophysical data in Table 1, which are 16.6\%, 4.2\%, 9.2\%, and 7.2\% for SI, DBSI, DBMI, and DIMI, respectively, derived from which, $\Phi_{\mathrm{p}}$ of $6.8 \%, 4.1 \%, 9.2 \%$, and $7.2 \%$ are obtained. Compared to those of SI crystals $\left(k_{r}^{p}=0.1 \mathrm{~s}^{-1}\right.$, and $\left.k_{n r}^{p}=1.7 \mathrm{~s}^{-1}\right)$, the presence of $\mathrm{Br}$ and I atoms do improve the RTP emission with enhanced radiative decay rates $\left(k_{r}^{p}, 2.5 \sim 29.0 \mathrm{~s}^{-1}\right)$, whereas the nonradiative decay rates $\left(k_{n r}^{p}\right.$, 127.9 695.6 $\left.\mathrm{s}^{-1}\right)$ are also strikingly increased, thus resulting in much shorter $\left\langle\tau>_{\mathrm{ps}}(1.38 \sim 7.67 \mathrm{~ms})\right.$. Moreover, the $\Phi_{\mathrm{p}}$ and RTP maxima could reach $9.2 \%$ and $745 \mathrm{~nm}$, respectively, which are rarely accessed even for classic aromatic phosphors. ${ }^{[8,20]}$ To the best of our knowledge, these are the first examples of nonconventional luminophores with diversified and efficient red/NIR RTP from well-defined single crystals, which would inspirit future construction of novel efficient RTP emitters with simple structure 
and fine control. Such efficient and diversified RTP could be rationalized by the CTE mechanism, namely, the clustering of these molecules results in effective TSC among nonconventional chromophores (e.g. imide, halogens), which afford clustered chromophores with enriched and lowered energy levels and narrowed energy gaps, thus beneficial for SOC and ISC transitions. ${ }^{[12,16]}$ Meanwhile, effective intermolecular interactions stiffened the cluster conformations, which stabilize triplets and bring about bright RTP emissions. ${ }^{[13-15]}$

It is also noted that from SI to DIMI crystals, the RTP is gradually bathochromically shifted, indicative of progressively expanded electron delocalization, which should be ascribed to the effective molecular clustering and halogen effects. And even red-shifted emission of DIMI compared to that of DBMI might be ascribed to the larger radius of I atom, which makes it more easily to share the lone pairs. Absorption of the crystals also suggests the same trend. While they all display absorptions at $\sim 205,250$, and $280 \mathrm{~nm}$, intensified and red-shifted bands are noticed for DBSI, DBMI, and DIMI crystals (Figure 2d), which should be associated with the halogen effects. To acquire further insights, solution photophysics were investigated. Taking DBSI for example, with increasing concentration, the PL turns on and the intensity is progressively enhanced, moreover, the emission color also evolves from blue $\left(10^{-4} \mathrm{~m}\right)$ to cyan $\left(10^{-2} \mathrm{~m}\right)$ and then to green $(0.1 \mathrm{~m})$, accompanying red-shifted PL maxima at 431, 466, and $496 \mathrm{~nm}$ (Figure 2e,f and S6). Corresponding absorption also depicts broad bands at high concentrations (Figure S7a), indicative of aggregation and extended conjugation. Particularly, for the $0.1 \mathrm{~m}$ solution, with $\lambda_{\text {ex }}$ varying from 330 to $420 \mathrm{~nm}$, $\lambda_{\mathrm{em}}$ changes from 490 to $530 \mathrm{~nm}$ (Figure S7b), demonstrating typical $\lambda_{\mathrm{ex}}$-dependent emission. ${ }^{[21]}$ Similar phenomena were also observed for the other solutions (Figure S8-S10), suggesting the formation of diverse emissive aggregates upon concentration, ${ }^{[21]}$ which is consistent with results of the crystals. 


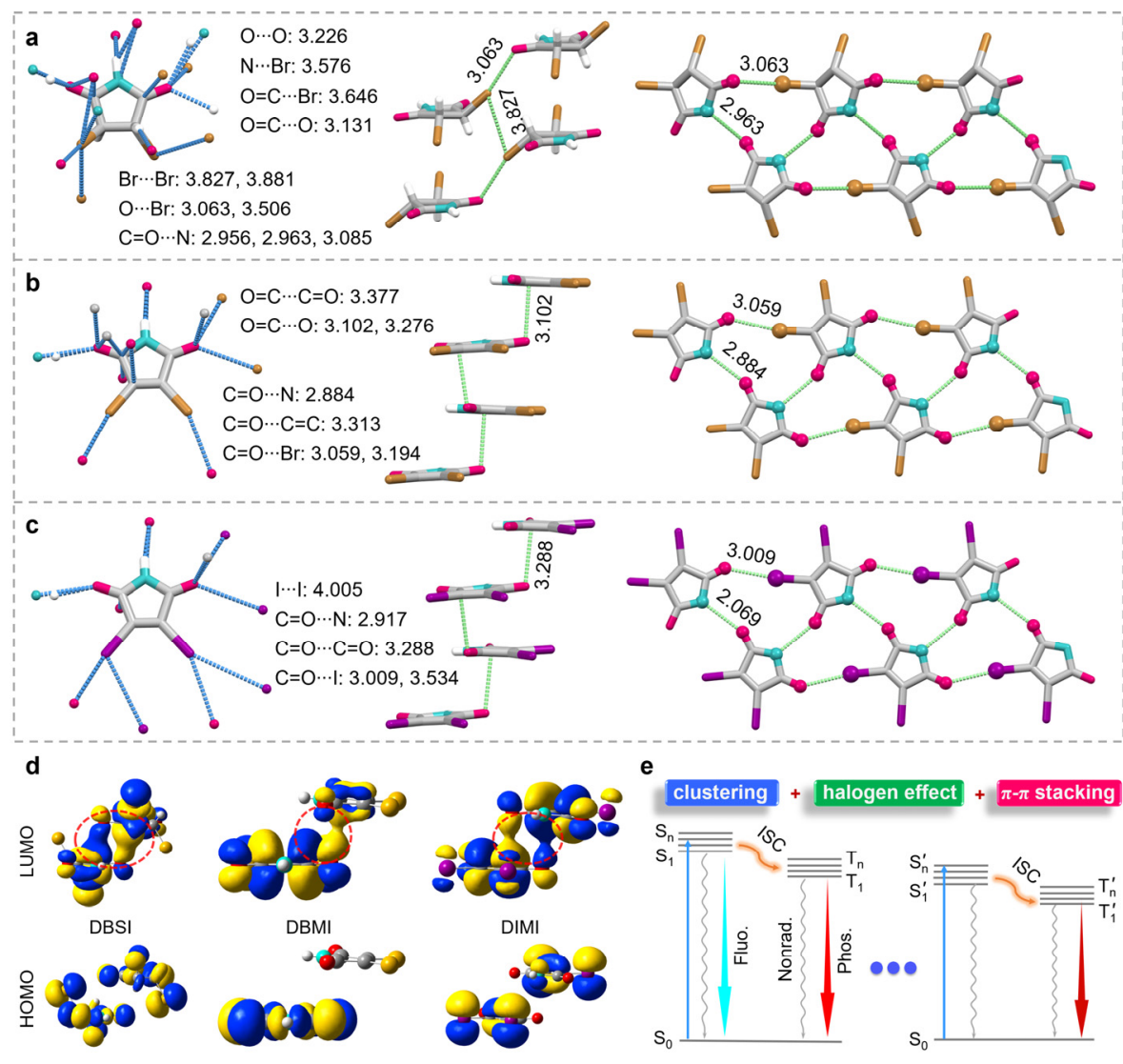

Figure 3. $(a-c)$ Single crystal structure with denoted intermolecular interactions and fragmental molecular packing with denoted short contacts among electron rich units of (a) DBSI, (b) DBMI, and (c) DIMI. (d) HOMO and LUMO electron densities of the selected dimers of DBSI, DBMI, and DIMI. (e) Demonstration of the luminescent mechanism and the generation of red/NIR RTP of the crystals.

Conformation and molecular packing are crucial to the solid luminescence, to gain further insights into the origin of the red/NIR RTP, single crystal structures of the compounds (Table S3) were examined. As shown in Figure 3a-c and S11, besides classic $\mathrm{C}=\mathrm{O} \cdots \mathrm{H}-\mathrm{N}$ hydrogen bonds and $\mathrm{C}=\mathrm{O} \cdots \mathrm{H}-\mathrm{C}$ interactions, $\mathrm{C}=\mathrm{O} \cdots \mathrm{C}=\mathrm{O}, \mathrm{C}=\mathrm{O} \cdots \mathrm{N}-\mathrm{H}$, and moreover $\mathrm{C}=\mathrm{O} \cdots \mathrm{X}(\mathrm{Br}$ and $\mathrm{I})$ short contacts are found in halogenated compounds. While all these noncovalent interactions readily rigidify the molecular conformations, the latter contacts among electron rich moieties significantly boost the TSCs, thus favoring much redder emissions. Notably, compared to SI, DBSI show similar molecular packing, while free of the planar hydrogen bonding dimers (Figure 1c) but with additional halogen contacts like $\mathrm{C}=\mathrm{O} \cdots \mathrm{Br}$ contacts $(3.063$ and $3.506 \AA$, Figure 3a), which implies the important role of halogen contacts in constructing effective and extended TSC, thanks to the sufficient electron delocalization between $\mathrm{Br}$ and other electron-rich 
groups. Furthermore, powerful $\pi-\pi$ stackings $(3.102,3.288 \AA)$ are present in DBMI and DIMI crystals, which should remarkably contribute to their red and NIR RTP. Specifically, considering the highly resemble molecular packings of DBMI and DIMI, their PL differences should be chiefly ascribed to the halogen atoms. Much larger radius and weaker electronegativity of I with comparison to those of $\mathrm{Br}$ enable better delocalization of the lone-pairs of I with other subunits, thus generating more apparently red-shifted PL. Consequent theoretical calculations of the HOMO and LUMO electron densities of certain dimers clearly show the TSC in crystals (Figure 3d), which agree with preceding results. Taken together, a preliminary conclusion could be drawn that molecular clustering, halogen effect, and $\pi-\pi$ stacking play significant synergistic roles in narrowing the energy gap, facilitating ISC process, and stiffening the conformations, thus resulting in unexpected multiple efficient red and NIR RTP emissions (Figure 3e).

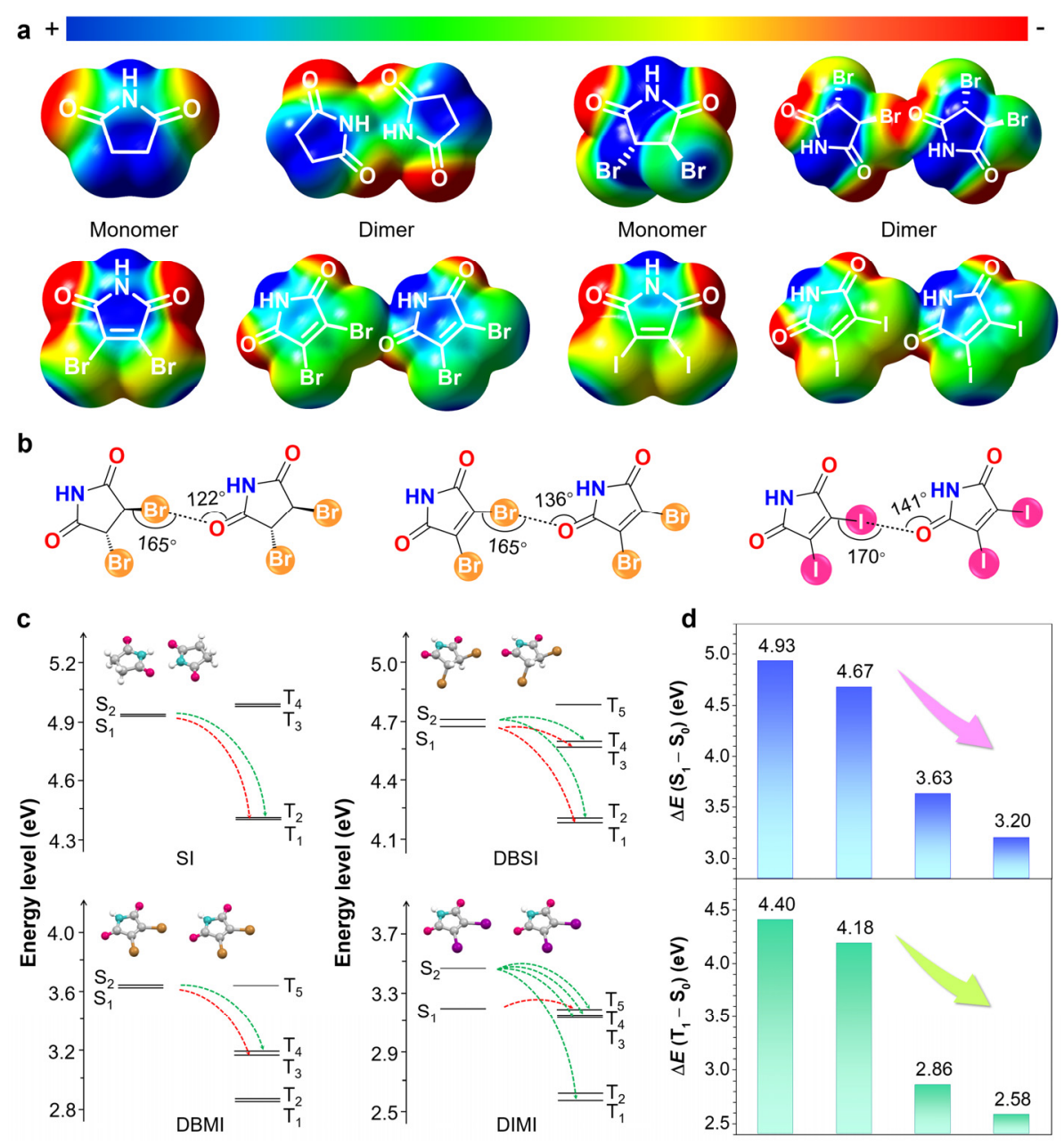


Figure 4. (a) Electric potential distributions of the HOMOs of monomers and dimers for varying compounds. (b) Illustration of the halogen contacts of DBSI, DBMI, and DIMI. (c) Energy levels of excited singlets and triplets of SI, DBSI, DBMI, and DIMI dimers. (d) Energy gaps of $\left(\mathrm{S}_{1}-\mathrm{S}_{0}\right)$ and $\left(\mathrm{T}_{1}-\mathrm{S}_{0}\right)$ for the dimers.

To theoretically probe the origin of effective TSCs in crystals, electric potential distributions of their monomer and dimer were calculated by the time-dependent density functional theory (TD-DFT) method. Remarkably, for the HOMO of SI, its negative charge is mainly located at the oxygen atoms of the carbonyls while the positive charge is distributed in the imide rings to construct the TSC (Figure 4a). After the introduction of halogen atoms $(\mathrm{Br}, \mathrm{I})$, which are ready to share their lone-pairs, $\sigma$-holes appear as acceptors to interact with the electrons from carbonyls (Figure 4a). Moreover, the distances of $\mathrm{C}=\mathrm{O} \cdots \mathrm{X}$ short contacts of three halogenated compounds are gradually shortened from 3.063 to $3.009 \AA$, and the $\mathrm{O} \cdots \mathrm{X}-\mathrm{C}$ bond angles are extended from $165^{\circ}$ to $170^{\circ}$ (Figure $4 \mathrm{~b}$ ), indicating gradually enhanced halogen contacts. Furthermore, from SI to DIMI, both energy gaps of $\left(\mathrm{S}_{1}-\mathrm{S}_{0}\right)$ and $\left(\mathrm{T}_{1}-\mathrm{S}_{0}\right)$ are continuously narrowed (Figure 4c,d and Table S4-S7), which well agrees with the progressively red-shifted trend in PL and RTP. These results again verify the collective consequence of clustering, halogen effect, and $\pi-\pi$ interaction in affording largely red-shifted emissions.

In summary, unexpected efficient red and NIR RTP are achieved in crystals of a group of halogenated SI derivatives devoid of considerable molecular conjugation, on account of the effectual molecular clustering, halogen effect, and $\pi-\pi$ stacking. Due to the concomitance of imide, $\mathrm{C}=\mathrm{C}$, halogen $(\mathrm{Br}, \mathrm{I})$ moieties, and the clustering of such groups, novel diversified clustered chromophores with stiffened conformations, remarkably extended delocalization, and boosted SOC and ISC are formed, thus enabling efficient multiple RTP emission in red and NIR regions. Notably, the electron sharing properties of halogens are highly important to the red-shifted RTP; meanwhile, the planar structure promoted $\pi$ - $\pi$ stacking further reinforces the bathochromic effect. This work implicates a rational way towards red and NIR phosphors through synergistic effect of planar structure, halogen effect, molecular clustering, and $\pi$ $\pi$ stacking, and may encourage future endeavors on nonconventional luminophores with tunable red and 
NIR emissions for emerging advanced applications. Furthermore, it reveals new aspects of halogen contacts $^{[22]}$ and provides in-depth mechanism understanding and rational regulation of the clusteroluminescence of nonconventional luminophores.

\section{Acknowledgement}

This work was financially supported by the National Natural Science Foundation of China (51822303, 52073172), the Natural Science Foundation of Shanghai (20ZR1429400), and the "Shuguang Program" (20SG11) of Shanghai Education Development Foundation and Shanghai Municipal Education Commission. The authors are grateful for the support for the PL and single crystal structure measurements by Dr. Ruibin Wang and Dr. Lingling Li at IAC of SJTU, respectively.

\section{References}

1. a) W. Zhao, Z. He, B. Z. Tang, Nat. Rev. Mater. 2020, 5, 869; b) S. Hirata, Adv. Opt. Mater. 2017, 5, 1700116; c) L. Xiao, H. Fu, Chem. Eur. J. 2019, 25, 714; d) W. Z. Yuan, X. Shen, H. Zhao, J. W. Y. Lam, L. Tang, P. Lu, C. Wang, Y. Liu, Z. Wang, Q. Zheng, J. Sun, Y. Ma, B. Z. Tang, J. Phys. Chem. C 2010, 114, 6090; e) N. Gan, H. Shi, Z. An, W. Huang, Adv. Funct. Mater. 2018, 28, 1802657.

2. T. Wang, X. Su, X. Zhang, X. Nie, L. Huang, X. Zhang, X. Sun, Y. Luo, G. Zhang, Adv. Mater. 2019, $31,1904273$.

3. a) S. M. A. Fateminia, Z. Mao, S. Xu, Z. Yang, Z. Chi, B. Liu, Angew. Chem. Int. Ed. 2017, 56, 12160; b) J. Yang, X. Zhen, B. Wang, X. Gao, Z. Ren, J. Wang, Y. Xie, J. Li, Q. Peng, K. Pu, Z. Li, Nat. Commun. 2018, 9, 840 .

4. G. Zhang, G. M. Palmer, M. Dewhirst, C. L. Fraser, Nat. Mater. 2009, 8, 747.

5. a) K. Jiang, Y. Wang, C. Cai, H. Lin, Adv. Mater. 2018, 30, 1800783; b) Y. Su, S. Z. F. Phua, Y. Li, X. Zhou, D. Jana, G. Liu, W. Q. Lim, W. K. Ong, C. Yang, Y. Zhao, Sci. Adv. 2018, 4, eaas9732.

6. a) Y. Lei, W. Dai, Y. Tian, J. Yang, P. Li, J. Shi, B. Tong, Z. Cai, Y. Dong, J. Phys. Chem. Lett. 2019, 10, 6019; b) Z. Y. Zhang, Y. Chen, Y. Liu, Angew. Chem. Int. Ed. 2019, 58, 6028. 
7. a) T. Zhang, X. Ma, H. Wu, L. Zhu, Y. Zhao, H. Tian, Angew. Chem. Int. Ed. 2020, 59, 11206; b) Z. Yang, Z. Mao, X. Zhang, D. Ou, Y. Mu, Y. Zhang, C. Zhao, S. Liu, Z. Chi, J. Xu, Y. Wu, P. Lu, A. Lien, M. R. Bryce, Angew. Chem. Int. Ed. 2016, 55, 2181; c) H. Li, H. Li, W. Wang, Y. Tao, S. Wang, Q. Yang, Y. Jiang, C. Zheng, W. Huang, R. Chen, Angew. Chem. Int. Ed. 2020, 59, 4756.

8. a) S. Hirata, K. Totani, J. Zhang, T. Yamashita, H. Kaji, S. R. Marder, T. Watanabe, C. Adachi, $A d v$. Funct. Mater. 2013, 23, 3386; b) O. Bolton, K. Lee, H. J. Kim, K. Y. Lin, J. Kim, Nat. Chem. 2011, 3, 205; c) Z. An, C. Zheng, Y. Tao, R. Chen, H. Shi, T. Chen, Z. Wang, H. Li, R. Deng, X. Liu, W. Huang, Nat. Mater. 2015, 14, 685; d) X. Wu, C. Y. Huang, D. G. Chen, D. Liu, C. Wu, K. J. Chou, B. Zhang, Y. Wang, Y. Liu, E. Y. Li, W. Zhu, P. T. Chou, Nat. Commun. 2020, 11, 2145.

9. a) Y. Su, Y. Zhang, Z. Wang, W. Gao, P. Jia, D. Zhang, C. Yang, Y. Li, Y. Zhao, Angew. Chem. Int. Ed. 2020, 59, 9967; b) X. F. Wang, H. Xiao, P. Z. Chen, Q. Z. Yang, B. Chen, C. H. Tung, Y. Z. Chen, L. Z. Wu, J. Am. Chem. Soc. 2019, 141, 5045; c) X. Chen, C. Xu, T. Wang, C. Zhou, J. Du, Z. Wang, H. Xu, T. Xie, G. Bi, J. Jiang, X. Zhang, J. N. Demas, C. O. Trindle, Y. Luo, G. Zhang, Angew. Chem. Int. Ed. 2016, 55, 9872; d) T. Zhang, X. Ma, H. Tian, Chem. Sci. 2020, 11, 482; e) W. Wang, Y. Zhang, W. J. Jin, Coordin. Chem. Rev. 2020, 404, 213107.

10. S. K. Lower, M. A. El-Sayed, Chem. Rev. 1966, 66, 199.

11. Y. Gong, Y. Tan, J. Mei, Y. Zhang, W. Z. Yuan, Y. Zhang, J. Z. Sun, B. Z. Tang, Sci. China Chem. 2013, 56, 1178 .

12. a) S. Zheng, T. Zhu, Y. Wang, T. Yang, W. Z. Yuan, Angew. Chem. Int. Ed. 2020, 59, 10018; b) Q. Zhou, T. Yang, Z. Zhong, F. Kausar, Z. Wang, Y. Zhang, W. Z. Yuan, Chem. Sci. 2020, 11, 2926; c) Y. Lai, T. Zhu, T. Geng, S. Zheng, T. Yang, Z. Zhao, G. Xiao, B. Zou, W. Z. Yuan, Small 2020, 16, 2005035.

13. a) M. Fang, J. Yang, X. Xiang, Y. Xie, Y. Dong, Q. Peng, Q. Li, Z. Li, Mater. Chem. Front. 2018, 2, 2124; b) Z. F. Liu, X. Chen, W. J. Jin, J. Mater. Chem. C 2020, 8, 7330; c) S. Wang, D. Wu, S. Yang, Z. Lin, Q. Ling, Mater. Chem. Front. 2020, 4, 1198. 
14. Y. Wang, S. Tang, Y. Wen, S. Zheng, B. Yang, W. Z. Yuan, Mater. Horiz. 2020, 7, 2105.

15. a) D. A. Tomalia, B. Klajnert-Maculewicz, K. A. M. Johnson, H. F. Brinkman, A. Janaszewska, D. M. Hedstrand, Prog. Polym. Sci. 2019, 90, 35; b) H. Zhang, Z. Zhao, P. R. McGonigal, R. Ye, S. Liu, J. W. Y. Lam, R. T. K. Kwok, W. Z. Yuan, J. Xie, A. L. Rogach, B. Z. Tang, Mater. Today 2020, 32, 275; c) B. Liu, H. Zhang, S. Liu, J. Z. Sun, X. H. Zhang, B. Z. Tang, Mater. Horiz. 2020, 7, 987; d) Y. Wang, Z. Zhao, W. Z. Yuan, ChemPlusChem 2020, 85, 1065.

16. Q. Zhou, B. Cao, C. Zhu, S. Xu, Y. Gong, W. Z. Yuan, Y. Zhang, Small 2016, 12, 6586.

17. H. Huang, Z. Chen, R. P. Ortiz, C. Newman, H. Usta, S. Lou, J. Youn, Y. Y. Noh, K. J. Baeg, L. X. Chen, A. Facchetti, T. J. Marks, J. Am. Chem. Soc. 2012, 134, 10966.

18. a) A. B. Mabire, M. P. Robin, W. D. Quan, H. Willcock, V. G. Stavros, R. K. O'Reilly, Chem. Commun. 2015, 51, 9733; b) B. He, J. Zhang, J. Zhang, H. Zhang, X. Wu, X. Chen, K. H. S. Kei, A. Qin, H. H. Y. Sung, J. W. Y. Lam, B. Z. Tang, Adv. Sci. 2021, 2004299.

19. The prompt emission spectra were measured and superposed by the data obtained from an ordinary detector (300 800 nm) and a NIR detector (500 1200 nm). See details in Supporting Information.

20. K. Kanosue, S. Ando, ACS Macro Lett. 2016, 5, 1301.

21. a) C. Shang, Y. Zhao, J. Long, Y. Ji, H. Wang, J. Mater. Chem. C 2020, 8, 1017; b) L. Bai, H. Yan, T. Bai, L. Guo, T. Lu, Y. Zhao, C. Li, Biomacromolecules 2020, 21, 3724; c) C. Hu, Y. Ru, Z. Guo, Z. Liu, J. Song, W. Song, X. Zhang, J. Qiao, J. Mater. Chem. C 2019, 7, 387.

22. G. Cavallo, P. Metrangolo, R. Milani, T. Pilati, A. Priimagi, G. Resnati, G. Terraneo, Chem. Rev. 2016, 116, 2478. 


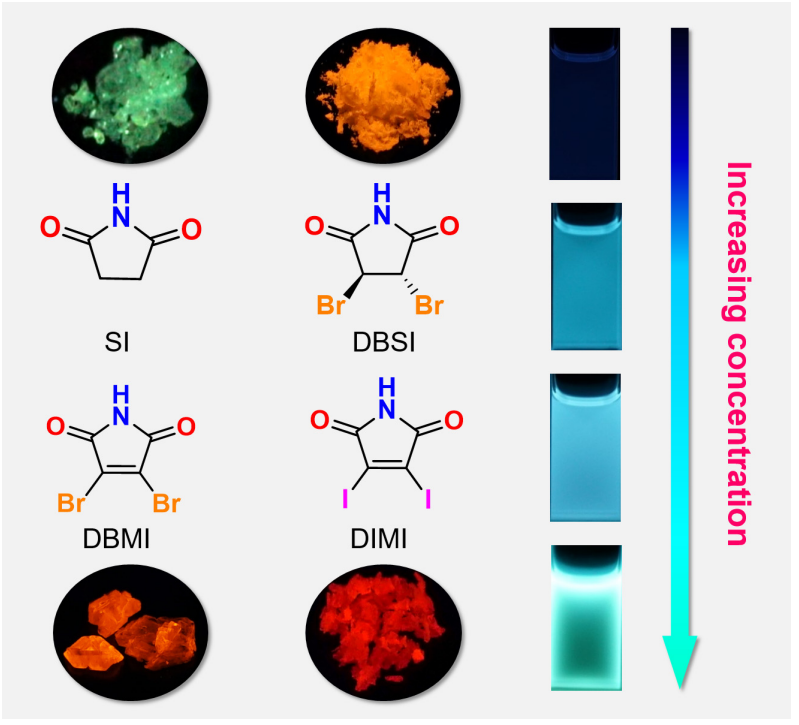

Small heterocycles, unexpected red/NIR phosphorescence. Despite rather limited molecular conjugation, the crystals of a group of five-membered cyclic imides demonstrate exceptional red and NIR room-temperature phosphorescence with high efficiencies and emission maxima of up to $9.2 \%$ and 750 $\mathrm{nm}$, respectively, on account of the extended through-space conjugation via clustering and halogen effects. 